Supporting information to:

Measuring metal uptake and loss in individual organisms: a novel double stable isotope method and its applications in explaining body size effects on cadmium concentration in mussels

Zhi Lin ${ }^{1}$, Xinyi Xu${ }^{1}$, Minwei Xie ${ }^{1,2}$, Rong Chen ${ }^{1,2}$, Qiao-Guo Tan*1,2

1. Fujian Provincial Key Laboratory for Coastal Ecology and Environmental Studies, College of the Environment and Ecology, Xiamen University, Xiamen, Fujian 361102, China

2. Center for Marine Environmental Chemistry and Toxicology, Xiamen University, Xiamen, Fujian 361102, China

*Corresponding Author: tanqg@xmu.edu.cn

Number of pages: 29

Number of Figures: 7 (Figure S1 to Figure S7)

Number of Tables: 4 (Table S1 to Table S4) 


\section{Table of Contents}

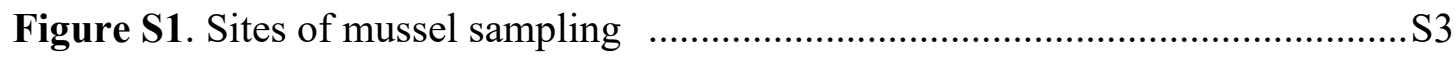

Figure S2. Relationship between soft tissue dry weight and shell length .................S4

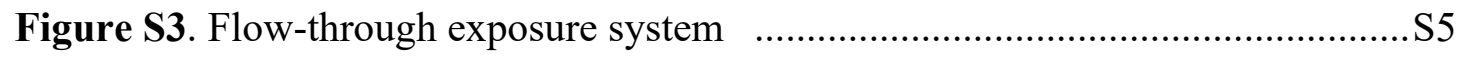

Figure S4. Comparison of toxicokinetic models based on metal mass and metal

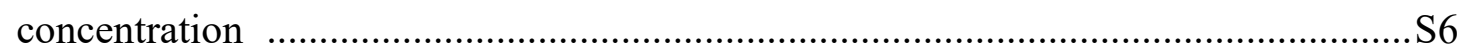

Figure S5. Soft tissue dry weight and shell length of mussels ................................S8

Figure S6. Concentration of newly accumulated ${ }^{113} \mathrm{Cd}$ and ${ }^{114} \mathrm{Cd}$ in mussels .......... S9

Figure S7. The goodness of fit of the toxicokinetic model ...................................S10

Table S1. Natural abundance of stable Cd isotopes ….........................................S1

Table S2. Literature $b$ values relating Cd concentration to body size ....................S12

Table S3. Literature $b$ values relating metal uptake rate to body size $\quad$...................S16

Table S4. Output of the multiple linear regression .............................................S18

Note S1. R code for estimating $k_{\mathrm{u}}$ and $k_{\mathrm{e}}$ from the double isotope data $\quad . . \ldots \ldots \ldots \ldots \ldots . . . . . . .519$ 


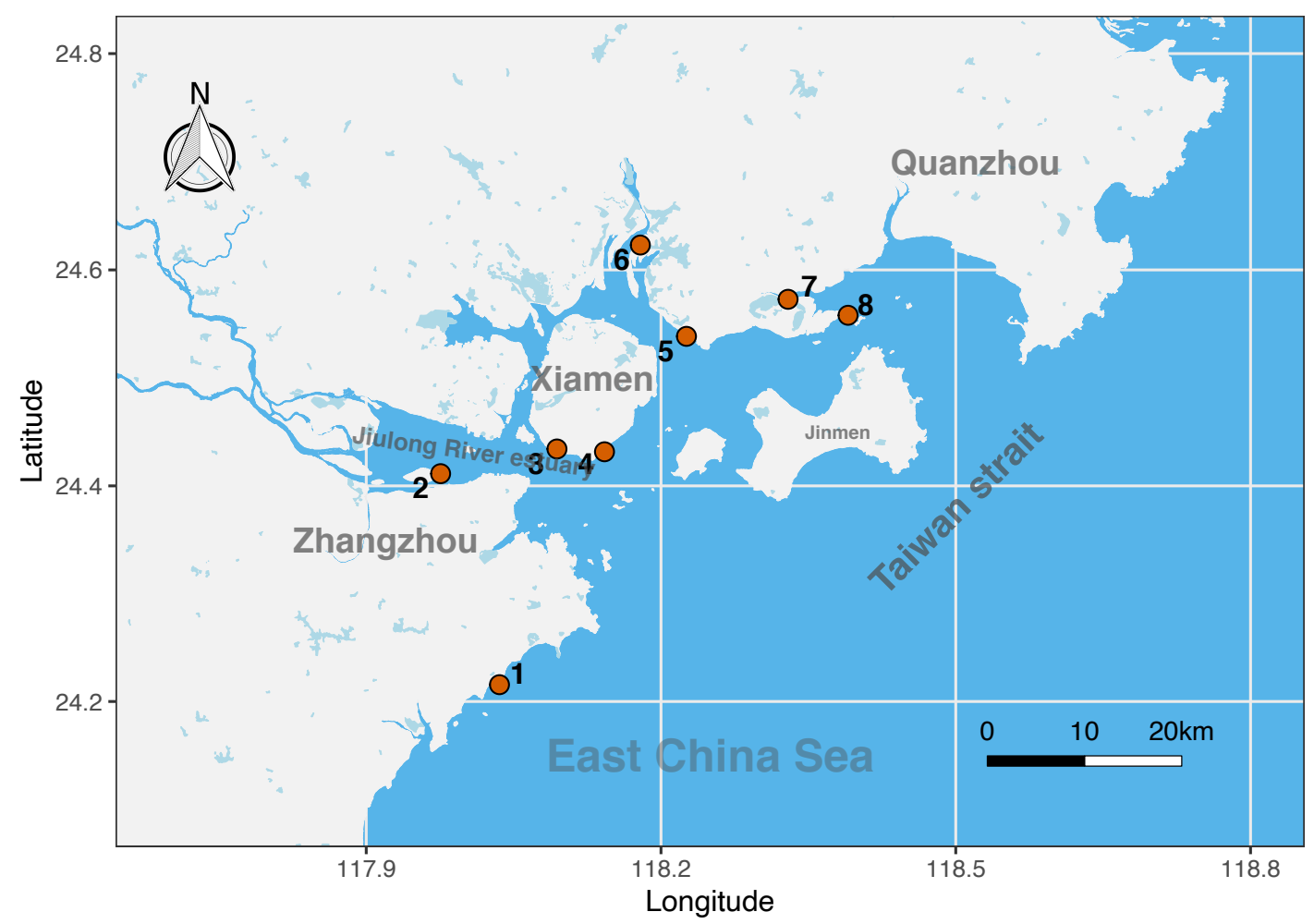

Figure S1. Sites of mussel sampling. The black-pigmy mussel $X$. atratus were sampled from 6 sites, including 2-Haimen Island $\left(117.9757^{\circ} \mathrm{E}, 24.4113^{\circ} \mathrm{N}\right), 3$ Baicheng $\left(118.1058^{\circ} \mathrm{E}, 24.4380^{\circ} \mathrm{N}\right)$, 4-Huangcuo $\left(118.1571^{\circ} \mathrm{E}, 24.4447^{\circ} \mathrm{N}\right), 5$ Aotou $\left(118.2269^{\circ} \mathrm{E}, 24.5416^{\circ} \mathrm{N}\right), 6$-Qiongtou $\left(118.1790^{\circ} \mathrm{E}, 24.6231^{\circ} \mathrm{N}\right)$, and 8Xiaodeng Island $\left(118.3904^{\circ} \mathrm{E}, 24.5580^{\circ} \mathrm{N}\right)$; the green mussel $P$. viridis were sampled from 2 sites, including 1-Baitang Bay $\left(118.0356^{\circ} \mathrm{E}, 24.2156^{\circ} \mathrm{N}\right)$ and 7-Dadeng Island $\left(118.3790^{\circ} \mathrm{E}, 24.5528^{\circ} \mathrm{N}\right)$. 
a

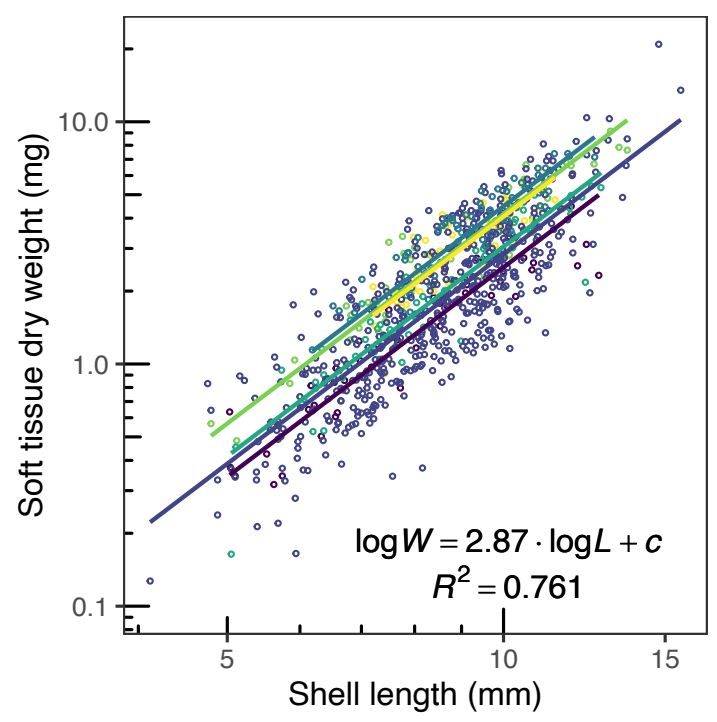

-2 - Haimen Island $\rightarrow 3$ - Baicheng

$\longrightarrow$ 4-Huangcuo $\rightarrow 5$ - Aotou

$=6$ - Qiongtou $\quad-\quad$ - Xiaodeng Island b $\quad P$. viridis

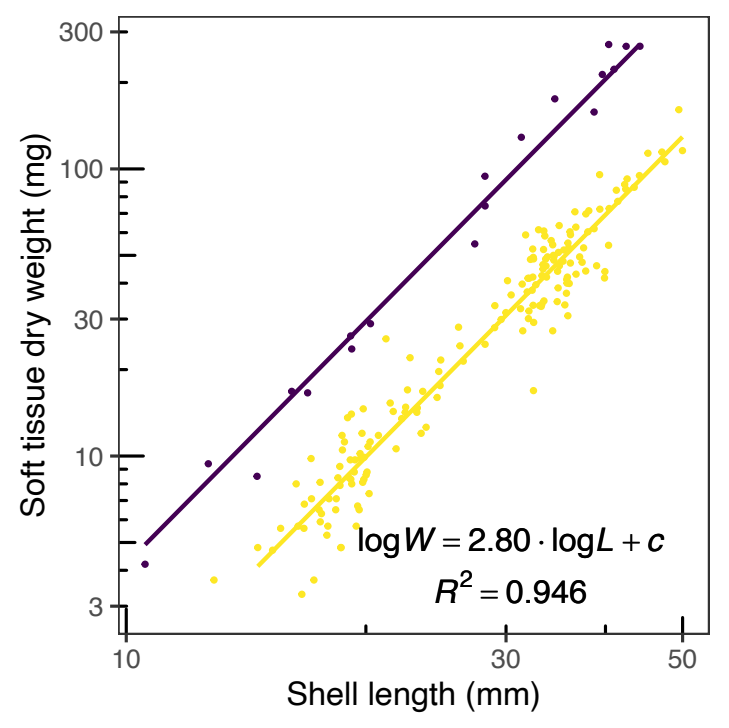

- 1 - Baitang Bay

7 - Dadeng Island

Figure S2. Linear relationship between soft tissue dry weight and shell length in

(a) X. atratus and (b) $P$. viridis on the double logarithmic scale. The intercept $c$ varies among sites: 2 -Hiamen Island $=-2.47,3$-Baicheng $=-2.42,4$-Huangcuo $=-$ 2.22, 5-Aotou $=-2.39,6$-Qiongtou $=-2.25,8$-Xiaodeng Island $=-2.26$; 1-Baitang Bay $=-5.18,7$-Dadeng Island $=-5.65$. 


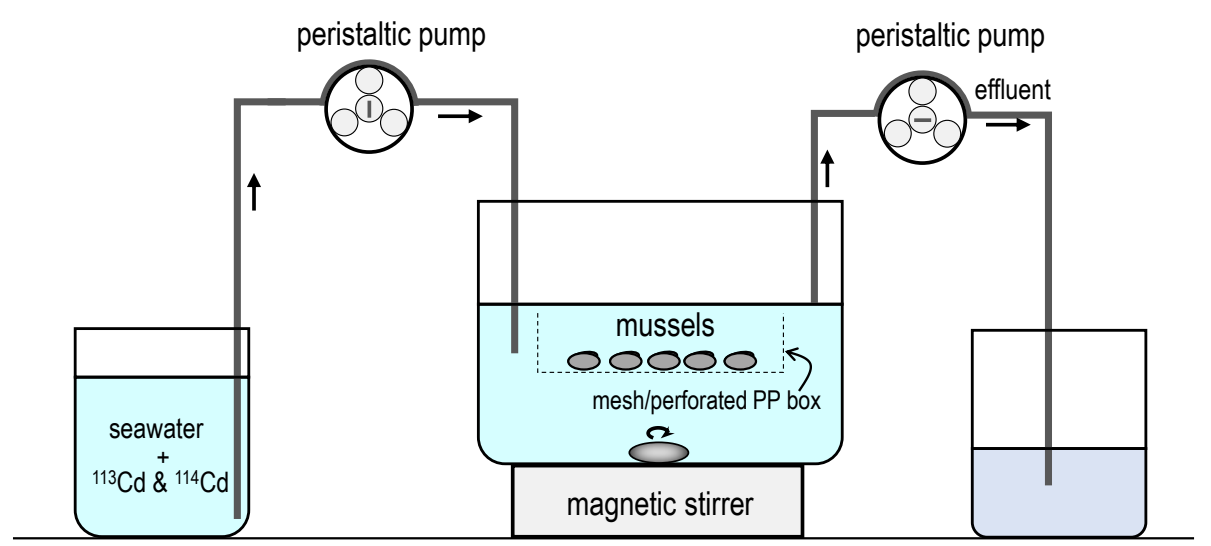

Figure S3. Flow-through exposure system. 


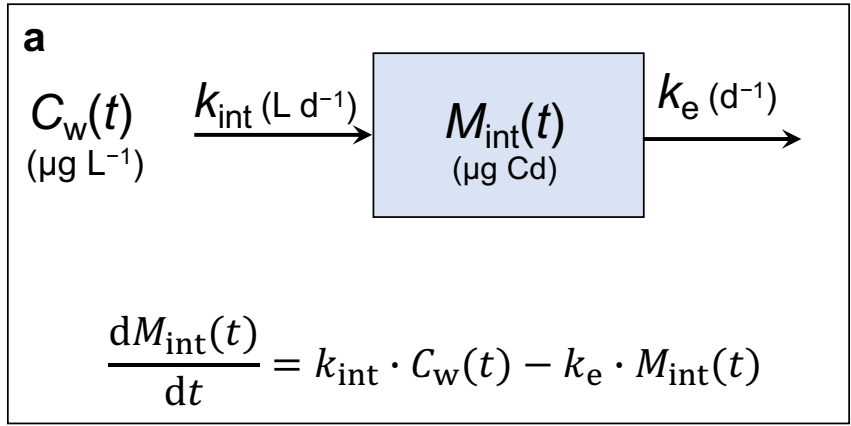

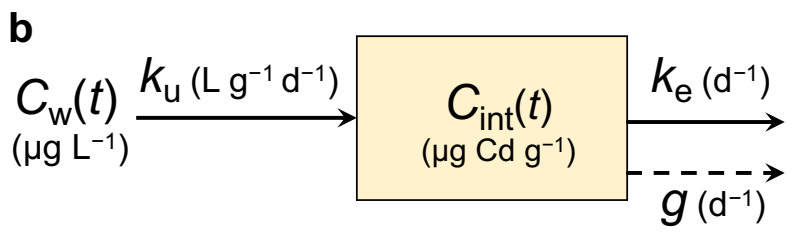

$$
\begin{aligned}
& \frac{\mathrm{d} C_{\mathrm{int}}(t)}{\mathrm{d} t}=k_{\mathrm{u}} \cdot C_{\mathrm{w}}(t)-\left(k_{\mathrm{e}}+g\right) \cdot C_{\mathrm{int}}(t)
\end{aligned}
$$

Figure S4. Comparison of the one-compartment toxicokinetic models based on (a) metal mass and (b) metal concentration in organisms. In this study, the model based on Cd mass (i.e., panel a) was used in the estimation of toxicokinetic parameters to avoid the need of estimating growth of organisms. The model based on $\mathrm{Cd}$ concentration (i.e., panel b) is more frequently used in the literature.

The two models can be converted into each other. According to the concept of mass balance, i.e., in a unit of time, mass of $\mathrm{Cd}$ accumulated by an organism $=$ mass of $\mathrm{Cd}$ entered the organism - mass of Cd eliminated from the organism, we get the mass-based equation (Figure S4a):

$$
\begin{gathered}
\frac{\mathrm{d} M_{\mathrm{in} t}(t)}{\mathrm{d} t}=k_{\mathrm{int}} \cdot C_{\mathrm{w}}(t)-k_{\mathrm{e}} \cdot M_{\mathrm{int}}(t) \\
M_{\mathrm{in} t}(t)=C_{\mathrm{in} t}(t) \cdot W(t)
\end{gathered}
$$

where $M_{\text {int }}=$ mass of Cd in mussels $(\mu \mathrm{g}), C_{\mathrm{w}}=$ concentration of $\mathrm{Cd}$ in exposure water $\left(\mu \mathrm{g} \mathrm{L}^{-1}\right), k_{\text {int }}=$ internalization rate constant $\left(\mathrm{L} \mathrm{d}^{-1}\right), k_{\mathrm{e}}=$ elimination rate constant $\left(\mathrm{d}^{-1}\right), C_{\text {int }}=$ concentration of $\mathrm{Cd}$ in mussels $\left(\mu \mathrm{g} \mathrm{g}^{-1}\right)$, and $W=$ dry weight of soft tissues of mussels (g). Substituting eq. S2 into eq. S1, we get:

$$
\frac{\mathrm{d}\left[C_{\mathrm{in} t}(t) \cdot W(t)\right]}{\mathrm{d} t}=k_{\mathrm{int}} \cdot C_{\mathrm{w}}(t)-k_{\mathrm{e}} \cdot C_{\mathrm{in} t}(t) \cdot W(t)
$$

According to the product rule, eq. S3 transforms to: 


$$
\frac{\mathrm{d} C_{\mathrm{in} t}(t)}{\mathrm{d} t} \cdot W(t)+\frac{\mathrm{d} W(t)}{\mathrm{d} t} \cdot C_{\mathrm{in} t}(t)=k_{\mathrm{int}} \cdot C_{\mathrm{w}}(t)-k_{\mathrm{e}} \cdot C_{\mathrm{in} t}(t) \cdot W(t)
$$

Assuming exponential growth of the mussels, we get:

$$
\frac{\mathrm{d} W(t)}{\mathrm{d} t}=g \cdot W(t)
$$

where $g=$ growth rate constant $\left(\mathrm{d}^{-1}\right)$. Substituting eq. S5 into eq. S4, we get:

$$
\frac{\mathrm{d} C_{\mathrm{in} t}(t)}{\mathrm{d} t} \cdot W(t)+g \cdot W(t) \cdot C_{\mathrm{in} t}(t)=k_{\mathrm{int}} \cdot C_{\mathrm{w}}(t)-k_{\mathrm{e}} \cdot C_{\mathrm{in} t}(t) \cdot W(t)
$$

Rearranging eq. S6, we get the concentration-based model (Figure S4b):

$$
\frac{\mathrm{d} C_{\mathrm{in} t}(t)}{\mathrm{d} t}=k_{\mathrm{u}} \cdot C_{\mathrm{w}}(t)-\left(k_{\mathrm{e}}+g\right) \cdot C_{\mathrm{in} t}(t)
$$

where $k_{\mathrm{u}}$ is the uptake rate constant $\left(\mathrm{L} \mathrm{g}^{-1} \mathrm{~d}^{-1}\right)$, and is defined as:

$$
k_{\mathrm{u}}=k_{\mathrm{int}} / W(t)
$$



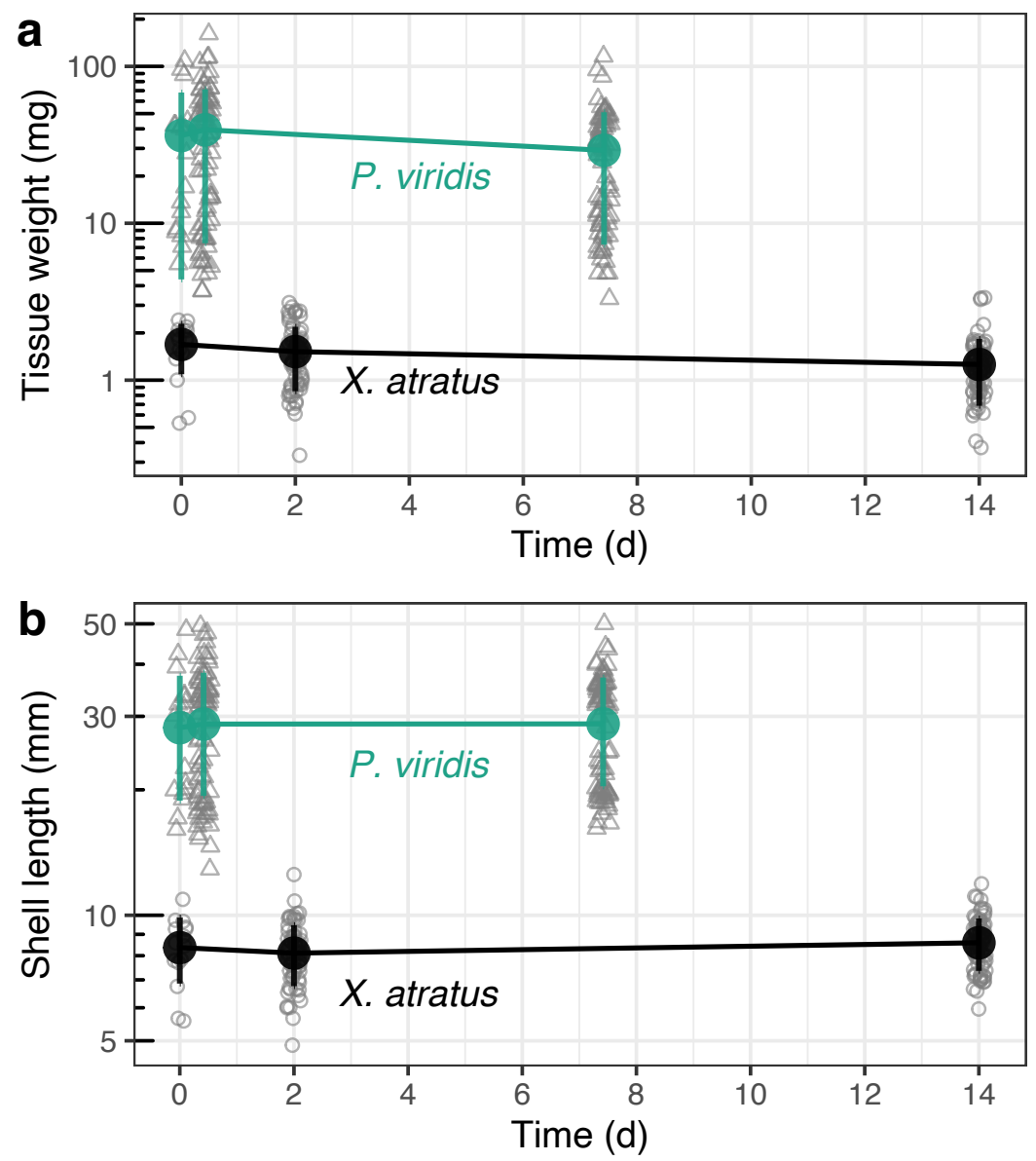

Figure S5. (a) Soft tissue dry weight and (b) shell length of $X$. atratus and $P$. viridis during the exposure. The solid points and error bars are mean values and standard deviations, respectively; the open symbols are individual measurements. 

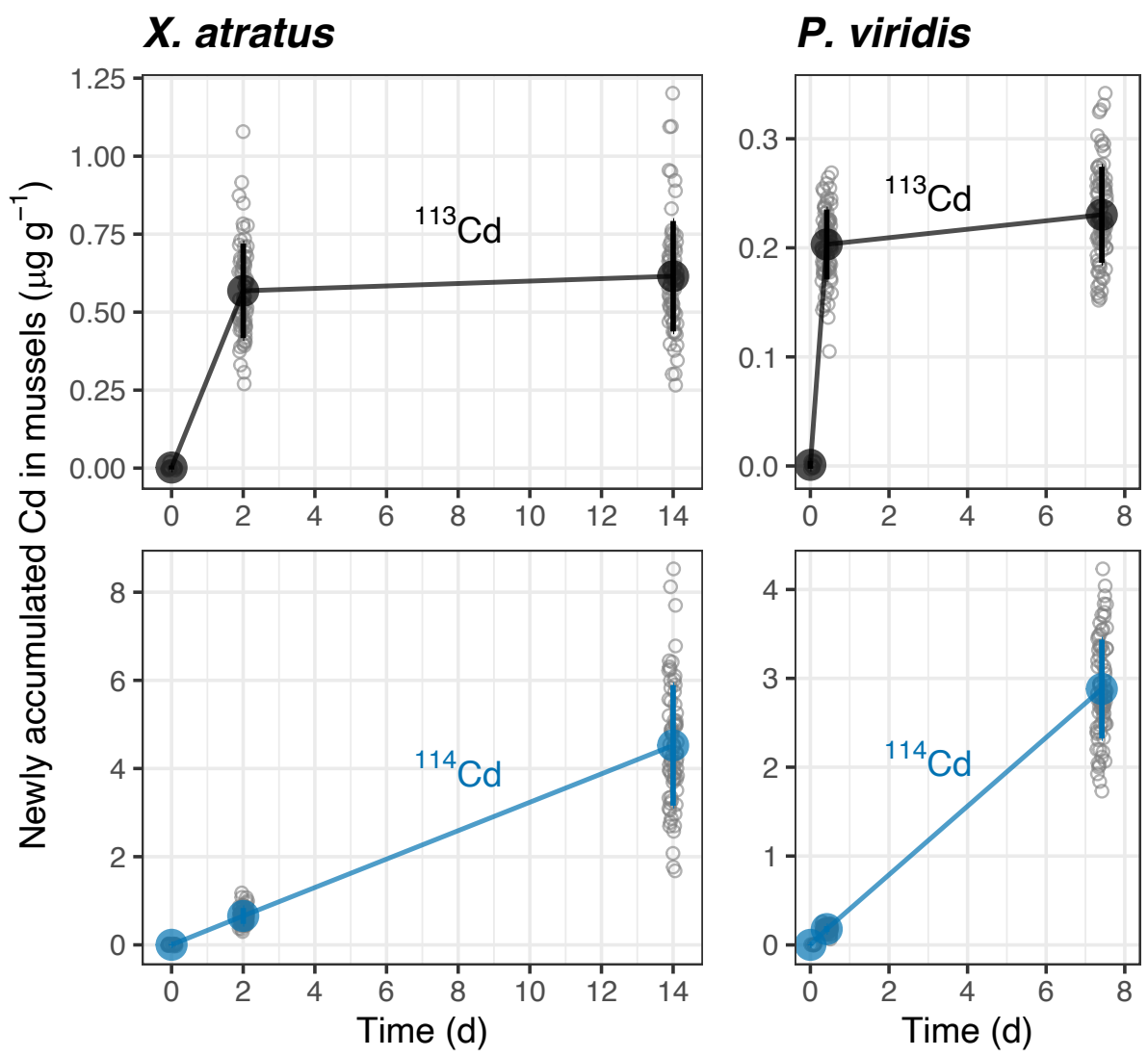

Figure S6. Concentrations of newly accumulated ${ }^{113} \mathrm{Cd}$ and ${ }^{114} \mathrm{Cd}$ in $\mathrm{X}$. atratus and $\boldsymbol{P}$. viridis during the exposure. Each open circle represents one individual mussel; the solid points and error bars are mean values and standard deviations, respectively.

It should be noted that concentrations of ${ }^{113} \mathrm{Cd}$ increased slightly, rather than decreased, during the second stage of exposure, although the mussels were not exposed to ${ }^{113} \mathrm{Cd}$ during that period. The slight increase was due to the decrease of tissue weight of the mussels (see Figure S5a) and not uptake of ${ }^{113} \mathrm{Cd}$. 

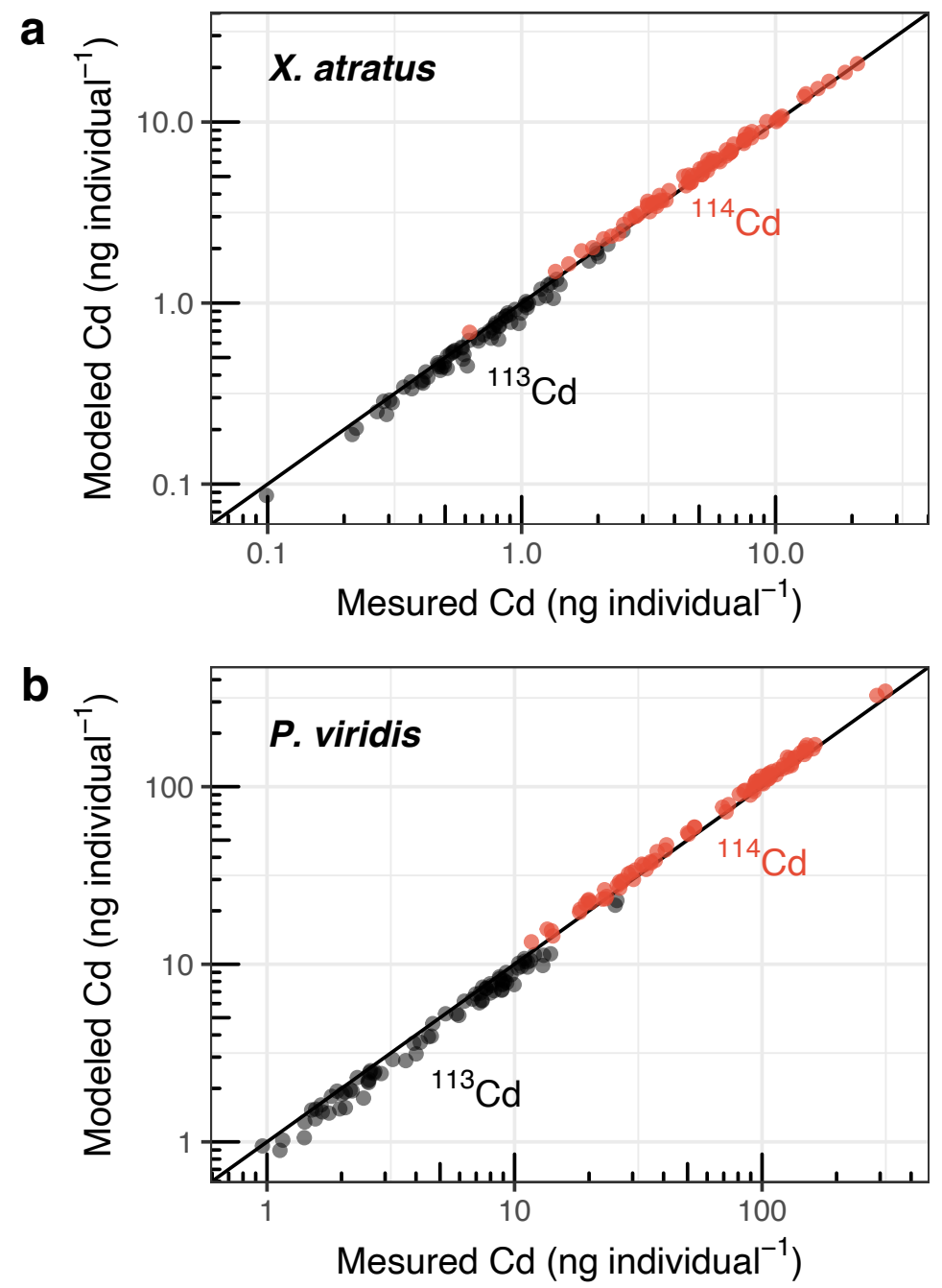

Figure S7. The goodness of fit of the one-compartment first-order toxicokinetic model to the Cd bioaccumulation in (a) X. atratus and (b) P. viridis. The points represent ${ }^{113} \mathrm{Cd}$ and ${ }^{114} \mathrm{Cd}$ content in mussel individuals; the solid lines are the 1:1 line. 
Table S1. Natural abundance of stable Cd isotopes and isotope composition of the enriched Cd isotope materials used in this study.

\begin{tabular}{lccc}
\hline Isotope & $\begin{array}{c}\text { Natural abundance } \\
(\%)\end{array}$ & $\begin{array}{c}\text { Enriched } \\
(\mathbf{\%})\end{array}$ & $\begin{array}{c}{ }^{113} \mathbf{C d} \\
(\%)\end{array}$ \\
\hline${ }^{106} \mathrm{Cd}$ & 1.25 & $<0.01$ & 0.005 \\
${ }^{108} \mathrm{Cd}$ & 0.89 & $<0.01$ & 0.005 \\
${ }^{110} \mathrm{Cd}$ & 12.49 & 0.13 & 0.005 \\
${ }^{111} \mathrm{Cd}$ & 12.8 & 0.18 & 0.005 \\
${ }^{112} \mathrm{Cd}$ & 24.13 & 1.63 & 0.005 \\
${ }^{113} \mathrm{Cd}$ & 12.22 & 94.8 & 0.365 \\
${ }^{114} \mathrm{Cd}$ & 28.73 & 3.03 & 99.07 \\
${ }^{116} \mathrm{Cd}$ & 7.49 & 0.23 & 0.54 \\
\hline
\end{tabular}

In this study, we selected ${ }^{113} \mathrm{Cd}$ and ${ }^{114} \mathrm{Cd}$ for the double stable isotope measurements. In theory, any two of them can be selected for experiment; nevertheless, there are several scientific and nonscientific factors need to be considered in selecting isotopes:

(1) the isotope is commercially available and economically affordable;

(2) the commercially available isotope has high enrichment level, e.g., 95\%, $99 \%$;

(3) the isotope of low relative natural abundance is preferred because it can produce higher sensitivity in chemical analysis;

(4) ICP-MS analysis of the isotope is not subject to substantial isobaric interferences. Although ${ }^{114} \mathrm{Cd}$ and ${ }^{113} \mathrm{Cd}$ are subject to the interference of ${ }^{114} \mathrm{Sn}$ (natural relative abundance $0.43 \%$ ) and ${ }^{113} \operatorname{In}$ (a rare element), respectively, the interferences are negligible due to the low abundance of ${ }^{114} \mathrm{Sn}$ and ${ }^{113} \mathrm{In}$. In this respect, ${ }^{116} \mathrm{Cd}$ may not be a good choice due to the interference from ${ }^{116} \mathrm{Sn}$ (natural relative abundance $14.53 \%$ ). 
Table S2. The $b$ values relating $\underline{C d}$ concentration in aquatic organisms to body size (expressed as dry tissue weight) on a double logarithmic scale. See eqs. 6 and 7 for the detailed definition of $b$.

\begin{tabular}{|c|c|c|c|c|}
\hline \multirow{2}{*}{ Common name } & \multicolumn{2}{|c|}{ Species name } & \multirow[t]{2}{*}{$b$ value } & \multirow[t]{2}{*}{ Reference } \\
\hline & used in reference & currently accepted & & \\
\hline Mussel & Perna viridis & Perna viridis & -0.126 & This study \\
\hline Mussel & Perna viridis & Perna viridis & -0.159 & This study \\
\hline Mussel & Xenostrobus atratus & Xenostrobus atratus & 0.348 & This study \\
\hline Mussel & Xenostrobus atratus & Xenostrobus atratus & 0.362 & This study \\
\hline Mussel & Xenostrobus atratus & Xenostrobus atratus & 0.177 & This study \\
\hline Mussel & Xenostrobus atratus & Xenostrobus atratus & 0.371 & This study \\
\hline Mussel & Xenostrobus atratus & Xenostrobus atratus & 0.416 & This study \\
\hline Mussel & Xenostrobus atratus & Xenostrobus atratus & 0.202 & This study \\
\hline Clam & Meretrix lusoria & Meretrix Iusoria & -0.254 & Ju et al., $2020^{[1]}$ \\
\hline Clam & Amiantis umbonella & Callista umbonella & 0.20 & Tarique et al., $2019^{[2]}$ \\
\hline Clam & Amiantis umbonella & Callista umbonella & -0.13 & Tarique et al., $2019^{[2]}$ \\
\hline Mussel & Dreissena polymorpha & Dreissena polymorpha & -0.582 & Tang et al., $2017^{[3]}$ \\
\hline Mussel & Perna viridis & Perna viridis & -0.588 & Yap et al., $2009^{[4]}$ \\
\hline Scallop & Chlamys nobilis & Mimachlamys crassicostata & -0.266 & Pan \& Wang, $2008^{[5]}$ \\
\hline Mussel & Mytilus galloprovincialis & Mytilus galloprovincialis & -0.234 & Conti et al., $2008^{[6]}$ \\
\hline Fish & Acanthopagrus schlegeli & Acanthopagrus schlegelii & -0.655 & Zhang \& Wang, $2007^{[7]}$ \\
\hline Clam & Gafrarium tumidum & Gafrarium pectinatum & $-0.38^{*}$ & Hédouin et al., $2006^{[8]}$ \\
\hline Mussel & Mytilus edulis & Mytilus edulis & -0.18 & Mubiana, $2006^{[9]}$ \\
\hline Mussel & Mytilus edulis & Mytilus edulis & -0.35 & Mubiana, $2006^{[9]}$ \\
\hline Snail & Monodonta turbinata & Phorcus turbinatus & 0.727 & Cubadda et al., $2001^{[10]}$ \\
\hline Snail & Monodonta mutabilis & Phorcus mutabilis & 0.766 & Cubadda et al., $2001^{[10]}$ \\
\hline
\end{tabular}




\begin{tabular}{|c|c|c|c|c|}
\hline \multirow{2}{*}{ Common name } & \multicolumn{2}{|c|}{ Species name } & \multirow[t]{2}{*}{$b$ value } & \multirow[t]{2}{*}{ Reference } \\
\hline & used in reference & currently accepted & & \\
\hline Limpet & Patella caerulea & Patella caerulea & 0.768 & Cubadda et al., $2001^{[10]}$ \\
\hline Limpet & Patella lusitanica & Patella rustica & 0.474 & Cubadda et al., $2001^{[10]}$ \\
\hline Snail & Nucella lapillus & Nucella lapillus & 0.31 & Leung et al., $2001^{[11]}$ \\
\hline Snail & Nucella lapillus & Nucella lapillus & -0.878 & Leung et al., $2001^{[11]}$ \\
\hline Snail & Nucella lapillus & Nucella lapillus & -0.055 & Leung et al., $2001^{[11]}$ \\
\hline Clam & Potamocorbula amurensis & Potamocorbula amurensis & -0.49 & Lee et al., $1998^{[12]}$ \\
\hline Clam & Macoma balthica & Limecola balthica & -0.48 & Lee et al., $1998^{[12]}$ \\
\hline Mussel & Mytilus edulis & Mytilus edulis & $0.272^{*}$ & Riget et al., $1996^{[13]}$ \\
\hline Mussel & Mytilus edulis & Mytilus edulis & $0.284^{*}$ & Riget et al., $1996^{[13]}$ \\
\hline Mussel & Mytilus edulis & Mytilus edulis & $0.168^{*}$ & Riget et al., $1996^{[13]}$ \\
\hline Mussel & Mytilus edulis & Mytilus edulis & $0.152^{*}$ & Riget et al., $1996^{[13]}$ \\
\hline Mussel & Mytilus edulis & Mytilus edulis & $0.108^{*}$ & Riget et al., $1996^{[13]}$ \\
\hline Mussel & Mytilus edulis & Mytilus edulis & $0.244^{*}$ & Riget et al., $1996^{[13]}$ \\
\hline Mussel & Mytilus edulis & Mytilus edulis & $0.18^{*}$ & Riget et al., $1996^{[13]}$ \\
\hline Mussel & Mytilus edulis & Mytilus edulis & $-0.08^{*}$ & Riget et al., $1996^{[13]}$ \\
\hline Mussel & Mytilus edulis & Mytilus edulis & $0.112^{*}$ & Riget et al., $1996^{[13]}$ \\
\hline Mussel & Mytilus edulis & Mytilus edulis & $0.324^{*}$ & Riget et al., $1996^{[13]}$ \\
\hline Mussel & Mytilus edulis & Mytilus edulis & $0.124^{*}$ & Riget et al., $1996^{[13]}$ \\
\hline Mussel & Mytilus edulis & Mytilus edulis & $0.188^{*}$ & Riget et al., $1996^{[13]}$ \\
\hline Snail & Monodonta turbinata & Phorcus turbinatus & $-0.288^{*}$ & Catsiki et al., $1994^{[14]}$ \\
\hline Snail & Monodonta turbinata & Phorcus turbinatus & $-0.188^{*}$ & Catsiki et al., $1994^{[14]}$ \\
\hline Snail & Cerithium vulgatum & Cerithium vulgatum & $-0.118^{*}$ & Catsiki et al., $1994^{[14]}$ \\
\hline Snail & Cerithium vulgatum & Cerithium vulgatum & $-0.020^{*}$ & Catsiki et al., $1994^{[14]}$ \\
\hline Clam & Arctica islandica & Arctica islandica & 0.51 & Swaileh \& Adelung, $1994^{[15]}$ \\
\hline
\end{tabular}




\begin{tabular}{|c|c|c|c|c|}
\hline \multirow{2}{*}{ Common name } & \multicolumn{2}{|c|}{ Species name } & \multirow[t]{2}{*}{$b$ value } & \multirow[t]{2}{*}{ Reference } \\
\hline & used in reference & currently accepted & & \\
\hline Clam & Astarte borealis & Astarte borealis & -0.094 & Fischer, $1983^{[16]}$ \\
\hline Clam & Astarte borealis & Astarte borealis & 0.425 & Fischer, $1983^{[16]}$ \\
\hline Cockle & Cardium edule & Cerastoderma edule & -0.247 & Fischer, $1983^{[16]}$ \\
\hline Cockle & Cardium edule & Cerastoderma edule & -0.282 & Fischer, $1983^{[16]}$ \\
\hline Clam & Mya arenaria & Mya arenaria & -0.020 & Fischer, $1983^{[16]}$ \\
\hline Clam & Macoma balthica & Limecola balthica & 0.028 & Fischer, $1983^{[16]}$ \\
\hline Mussel & Mytilus edulis & Mytilus edulis & 0.029 & Fischer, $1983^{[16]}$ \\
\hline Snail & Littorina littorea & Littorina littorea & 0.047 & Fischer, $1983^{[16]}$ \\
\hline Mussel & Mytilus edulis & Mytilus edulis & -0.31 & Cossa et al., $1980^{[17]}$ \\
\hline Mussel & Mytilus edulis & Mytilus edulis & -0.44 & Cossa et al., $1980^{[17]}$ \\
\hline Mussel & Mytilus edulis & Mytilus edulis & -0.45 & Cossa et al., $1980^{[17]}$ \\
\hline Mussel & Mytilus edulis & Mytilus edulis & -0.69 & Cossa et al., $1980^{[17]}$ \\
\hline Mussel & Mytilus edulis & Mytilus edulis & -0.34 & Cossa et al., $1980^{[17]}$ \\
\hline Mussel & Mytilus edulis & Mytilus edulis & -0.29 & Cossa et al., $1979^{[18]}$ \\
\hline Mussel & Mytilus edulis & Mytilus edulis & -0.35 & Cossa et al., $1979^{[18]}$ \\
\hline Mussel & Mytilus edulis & Mytilus edulis & -0.31 & Cossa et al., $1979^{[18]}$ \\
\hline Mussel & Mytilus edulis & Mytilus edulis & -0.54 & Cossa et al., $1979^{[18]}$ \\
\hline Oyster & Ostrea edulis & Ostrea edulis & -0.06 & Boyden, $1977^{[19]}$ \\
\hline Oyster & Ostrea edulis & Ostrea edulis & -0.04 & Boyden, $1977^{[19]}$ \\
\hline Oyster & Crassostrea gigas & Magallana gigas & -0.15 & Boyden, $1977^{[19]}$ \\
\hline Oyster & Crassostrea gigas & Magallana gigas & -0.14 & Boyden, $1977^{[19]}$ \\
\hline Oyster & Crassostrea gigas & Magallana gigas & -0.15 & Boyden, $1977^{[19]}$ \\
\hline Mussel & Mytilus edulis & Mytilus edulis & -0.03 & Boyden, $1977^{[19]}$ \\
\hline Mussel & Mytilus edulis & Mytilus edulis & 0.05 & Boyden, $1977^{[19]}$ \\
\hline
\end{tabular}




\begin{tabular}{|c|c|c|c|c|}
\hline \multirow{2}{*}{ Common name } & \multicolumn{2}{|c|}{ Species name } & \multirow[t]{2}{*}{$b$ value } & \multirow[t]{2}{*}{ Reference } \\
\hline & used in reference & currently accepted & & \\
\hline Mussel & Mytilus edulis & Mytilus edulis & 0.02 & Boyden, $1977^{[19]}$ \\
\hline Mussel & Mytilus edulis & Mytilus edulis & 0.08 & Boyden, $1977^{[19]}$ \\
\hline Mussel & Mytilus edulis & Mytilus edulis & -0.05 & Boyden, $1977^{[19]}$ \\
\hline Clam & Mercenaria mercenaria & Mercenaria mercenaria & -0.19 & Boyden, $1977^{[19]}$ \\
\hline Clam & Venerupis decussat & Ruditapes decussatus & -0.23 & Boyden, $1977^{[19]}$ \\
\hline Scallop & Chlamys opercular & Aequipecten opercularis & -0.04 & Boyden, $1977^{[19]}$ \\
\hline Snail & Littorina littorea & Littorina littorea & -0.03 & Boyden, $1977^{[19]}$ \\
\hline Snail & Buccinum undatum & Buccinum undatum & 0.18 & Boyden, $1977^{[19]}$ \\
\hline Snail & Scaphander lignarius & Scaphander lignarius & -0.06 & Boyden, $1977^{[19]}$ \\
\hline Limpet & Crepidula fornicata & Crepidula fornicata & 0.12 & Boyden, $1977^{[19]}$ \\
\hline Limpet & Patella intermedia & Patella depressa & 0.35 & Boyden, $1977^{[19]}$ \\
\hline Limpet & Patella intermedia & Patella depressa & 0.49 & Boyden, $1977^{[19]}$ \\
\hline Limpet & Patella vulgata & Patella vulgata & 1.05 & Boyden, $1977^{[19]}$ \\
\hline Limpet & Patella vulgata & Patella vulgata & 0.98 & Boyden, $1977^{[19]}$ \\
\hline Limpet & Patella vulgata & Patella vulgata & 0.96 & Boyden, $1977^{[19]}$ \\
\hline Limpet & Patella vulgata & Patella vulgata & 0.70 & Boyden, $1977^{[19]}$ \\
\hline Limpet & Patella vulgata & Patella vulgata & 0.37 & Boyden, $1977^{[19]}$ \\
\hline Oyster & Crassostrea gigas & Magallana gigas & -0.208 & Watling \& Watling, $1976^{[20]}$ \\
\hline Oyster & Crassostrea margaritacea & Striostrea margaritacea & -0.111 & Watling \& Watling, $1976^{[20]}$ \\
\hline Oyster & Ostrea edulis & Ostrea edulis & -0.355 & Watling \& Watling, $1976^{[20]}$ \\
\hline Cockle & Cerastoderma edule & Cerastoderma edule & -0.25 & Boyden, $1974^{[21]}$ \\
\hline
\end{tabular}

* Body size was expressed as shell length (or width); the listed $b$ values are the original $b$ values divided by a factor of 2.5 , assuming that body weight is proportional to shell length (or width) with an exponent of 2.5. 
Table S3. The $b$ values relating metal uptake rate in aquatic organisms to body size (expressed as dry tissue weight) on a double logarithmic scale. See eqs. 6 and 7 for the detailed definition of $b$.

\begin{tabular}{|c|c|c|c|c|c|}
\hline \multirow[t]{2}{*}{ Common name } & \multicolumn{2}{|c|}{ Species name } & \multirow[t]{2}{*}{ Metal } & \multirow[t]{2}{*}{$b$ value } & \multirow[t]{2}{*}{ Reference } \\
\hline & used in reference & currently accepted & & & \\
\hline Mussel & Perna viridis & Perna viridis & $\mathrm{Cd}$ & -0.072 & This study \\
\hline Mussel & Xenostrobus atratus & Xenostrobus atratus & $\mathrm{Cd}$ & 0.310 & This study \\
\hline Mussel & Dreissena polymorpha & Dreissena polymorpha & $\mathrm{Cd}$ & -0.379 & Tang et al., $2017^{[3]}$ \\
\hline Fish & Acanthopagrus schlegeli schlegeli & Acanthopagrus schlegelii & $\mathrm{Hg}$ & -0.68 & Dang \& Wang, $2012^{[22]}$ \\
\hline Fish & Acanthopagrus schlegeli schlegeli & Acanthopagrus schlegelii & $\mathrm{MeHg}$ & -0.54 & Dang \& Wang, 2012 ${ }^{[22]}$ \\
\hline Scallop & Chlamys nobilis & Mimachlamys crassicostata & $\mathrm{Cd}$ & -0.259 & Pan \& Wang, $2008^{[5]}$ \\
\hline Scallop & Chlamys nobilis & Mimachlamys crassicostata & $\mathrm{Zn}$ & -0.266 & Pan \& Wang, $2008^{[5]}$ \\
\hline Fish & Acanthopagrus schlegeli & Acanthopagrus schlegelii & $\mathrm{Cd}$ & -0.662 & Zhang \& Wang, $2007^{[7]}$ \\
\hline Fish & Acanthopagrus schlegeli & Acanthopagrus schlegelii & $\mathrm{Se}$ & -0.619 & Zhang \& Wang, $2007^{[7]}$ \\
\hline Fish & Acanthopagrus schlegeli & Acanthopagrus schlegelii & $\mathrm{Zn}$ & -0.615 & Zhang \& Wang, $2007^{[7]}$ \\
\hline Mussel & Perna viridis & Perna viridis & $\mathrm{Cd}$ & -0.594 & Chong \& Wang, $2001^{[23]}$ \\
\hline Clam & Ruditapes philippinarum & Ruditapes philippinarum & $\mathrm{Cd}$ & -0.56 & Chong \& Wang, $2001^{[23]}$ \\
\hline Mussel & Perna viridis & Perna viridis & $\mathrm{Cr}$ & -0.041 & Chong \& Wang, $2001^{[23]}$ \\
\hline Clam & Ruditapes philippinarum & Ruditapes philippinarum & $\mathrm{Cr}$ & -0.477 & Chong \& Wang, $2001^{[23]}$ \\
\hline Mussel & Perna viridis & Perna viridis & $\mathrm{Zn}$ & -0.399 & Chong \& Wang, $2001^{[23]}$ \\
\hline Clam & Ruditapes philippinarum & Ruditapes philippinarum & $\mathrm{Zn}$ & -0.503 & Chong \& Wang, $2001^{[23]}$ \\
\hline Mussel & Perna viridis & Perna viridis & Cs & -0.464 & Ke et al., $2000^{[24]}$ \\
\hline
\end{tabular}




\begin{tabular}{|c|c|c|c|c|c|}
\hline \multirow[t]{2}{*}{ Common name } & \multicolumn{2}{|c|}{ Species name } & \multirow[t]{2}{*}{ Metal } & \multirow[t]{2}{*}{$b$ value } & \multirow[t]{2}{*}{ Reference } \\
\hline & used in reference & currently accepted & & & \\
\hline Mussel & Septifer virgatus & Mytilisepta virgata & $\mathrm{Cd}$ & -0.537 & Wang \& Dei, $1999^{[25]}$ \\
\hline Mussel & Septifer virgatus & Mytilisepta virgata & $\mathrm{Cr}$ & -0.344 & Wang \& Dei, $1999^{[25]}$ \\
\hline Mussel & Septifer virgatus & Mytilisepta virgata & $\mathrm{Se}$ & -0.317 & Wang \& Dei, $1999^{[25]}$ \\
\hline Mussel & Septifer virgatus & Mytilisepta virgata & $\mathrm{Zn}$ & -0.437 & Wang \& Dei, $1999^{[25]}$ \\
\hline Clam & Potamocorbula amurensis & Potamocorbula amurensis & $\mathrm{Cd}$ & -0.27 & Lee et al., $1998^{[12]}$ \\
\hline Clam & Macoma balthica & Limecola balthica & $\mathrm{Cd}$ & -0.43 & Lee et al., $1998^{[12]}$ \\
\hline Clam & Potamocorbula amurensis & Potamocorbula amurensis & $\mathrm{Cr}$ & -0.05 & Lee et al., $1998^{[12]}$ \\
\hline Clam & Macoma balthica & Limecola balthica & $\mathrm{Cr}$ & -0.34 & Lee et al., $1998^{[12]}$ \\
\hline Clam & Potamocorbula amurensis & Potamocorbula amurensis & $\mathrm{Zn}$ & -0.23 & Lee et al., $1998^{[12]}$ \\
\hline Clam & Macoma balthica & Limecola balthica & $\mathrm{Zn}$ & -0.35 & Lee et al., $1998^{[12]}$ \\
\hline Fish & Gambusia affinis & Gambusia affinis & $\mathrm{Zn}$ & -0.90 & Newman \& Mitz, $1988^{[26]}$ \\
\hline
\end{tabular}


Table S4. Output of the multiple linear regression indicates that $k_{\mathrm{u}}$ was the significant predictor of $\mathrm{Cd}$ concentrations in $X$. atratus and $P$. viridis while organism weight was not. The regression model is

$$
\log C d=\beta_{0}+\beta_{1} \cdot \log k_{\mathrm{u}}+\beta_{2} \cdot \log W
$$

where $C d$ is the background Cd concentration in the mussels at the end of the exposure (i.e., T2), $k_{\mathrm{u}}$ is the uptake rate constant of $\mathrm{Cd}$, and $W$ is the dry weight of soft tissues.

(a) Mussel $X$. atratus:

\begin{tabular}{lllll}
\hline & Estimate & Standard error & $t$ value & $p$ value \\
\hline Intercept & 1.47621 & 0.25270 & 5.842 & $1.38 \mathrm{e}-07$ \\
$\log \left(k_{\mathrm{u}}\right)$ & 0.32856 & 0.12078 & 2.720 & $\mathbf{0 . 0 0 8 1 7}$ \\
$\log (W)$ & 0.18574 & 0.09365 & 1.983 & 0.05115 \\
\hline
\end{tabular}

(b) Mussel $P$. viridis:

\begin{tabular}{lllll}
\hline & Estimate & Standard error & $t$ value & $p$ value \\
\hline Intercept & 0.70280 & 0.07929 & 8.864 & $2.19 \mathrm{e}-13$ \\
$\log \left(k_{\mathrm{u}}\right)$ & 0.76497 & 0.09602 & 7.967 & $1.18 \mathrm{e}-11$ \\
$\log (W)$ & -0.03409 & 0.02115 & -1.612 & 0.111 \\
\hline
\end{tabular}


Note S1. R code for estimating the toxicokinetic parameters $\left(k_{\mathrm{u}}\right.$ and $\left.\boldsymbol{k}_{\mathrm{e}}\right)$

$\mathrm{R}$ code in text format is also available at: https://github.com/tan-qiaoguo/double_stable isotope

library(deSolve) \# for using the function "ode" to integrate library(FME) \# for using the function "modFit" to fit library(readxl) \# for importing data from Excel files library(ggplot2) \# for plotting library(dplyr) \# for manipulating data frames

\section{Cd isotope concentrations in exposure solution}

The data on $\mathrm{Cd}$ isotope concentrations in exposure solution are imported from the Excel file provided as another Supporting Information document. The *.xlsx file name in the code below should be revised to be the same as the that of the Excel file.

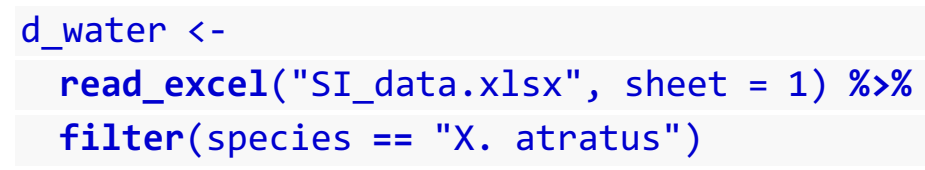

\section{Cd isotope concentrations in mussels}

Similarly, the data on the Cd isotope concentrations in the mussels during the double isotope exposure are imported from the same Excel file mentioned above but from a different sheet.

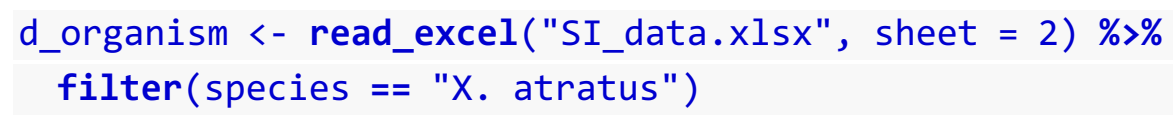

\section{Time points}

In the double Cd isotope exposure of $X$. atratus, $\mathrm{T} 1$ and $\mathrm{T} 2$ were $48 \mathrm{~h}$ and $336 \mathrm{~h}$, respectively.

$\mathrm{T} 1<-48 \# T 1=48 \mathrm{~h}$

T2<- $336 \#$ T2 $=336 h$ 
The total exposure length (i.e., T2 $=336 \mathrm{~h}$ ) is divided into intervals of $1 / 48 \mathrm{~d}$ (i.e., $0.5 \mathrm{~h}$ ). The time sequence is used below to interpolate the exposure concentration and to calculate the bioaccumulation of $\mathrm{Cd}$ isotopes.

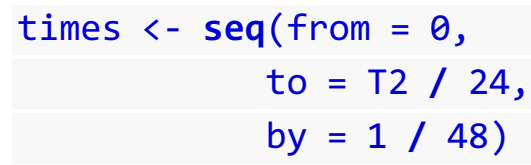

\section{Interpolation of $\mathrm{Cd}$ isotope concentrations in solutions}

Cd isotope concentrations in the exposure solutions during the whole experiment period was interpolated from the measured concentrations using the function approxfun.

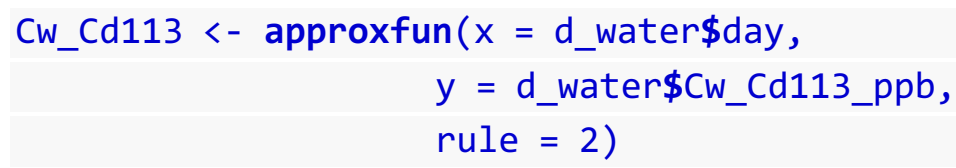

The interpolated concentrations of ${ }^{113} \mathrm{Cd}$ are shown below:
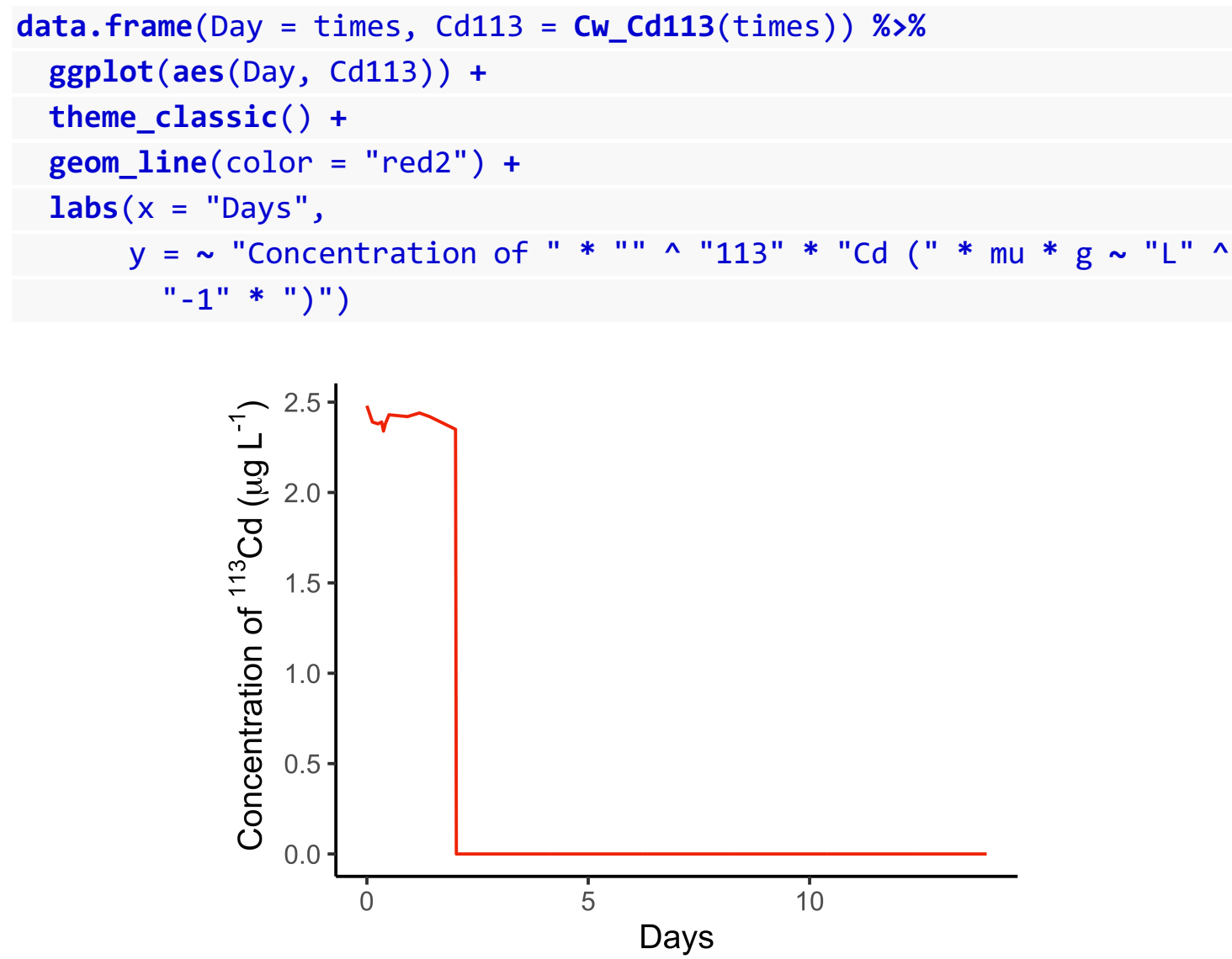
The interpolated concentrations of ${ }^{114} \mathrm{Cd}$ are shown below:
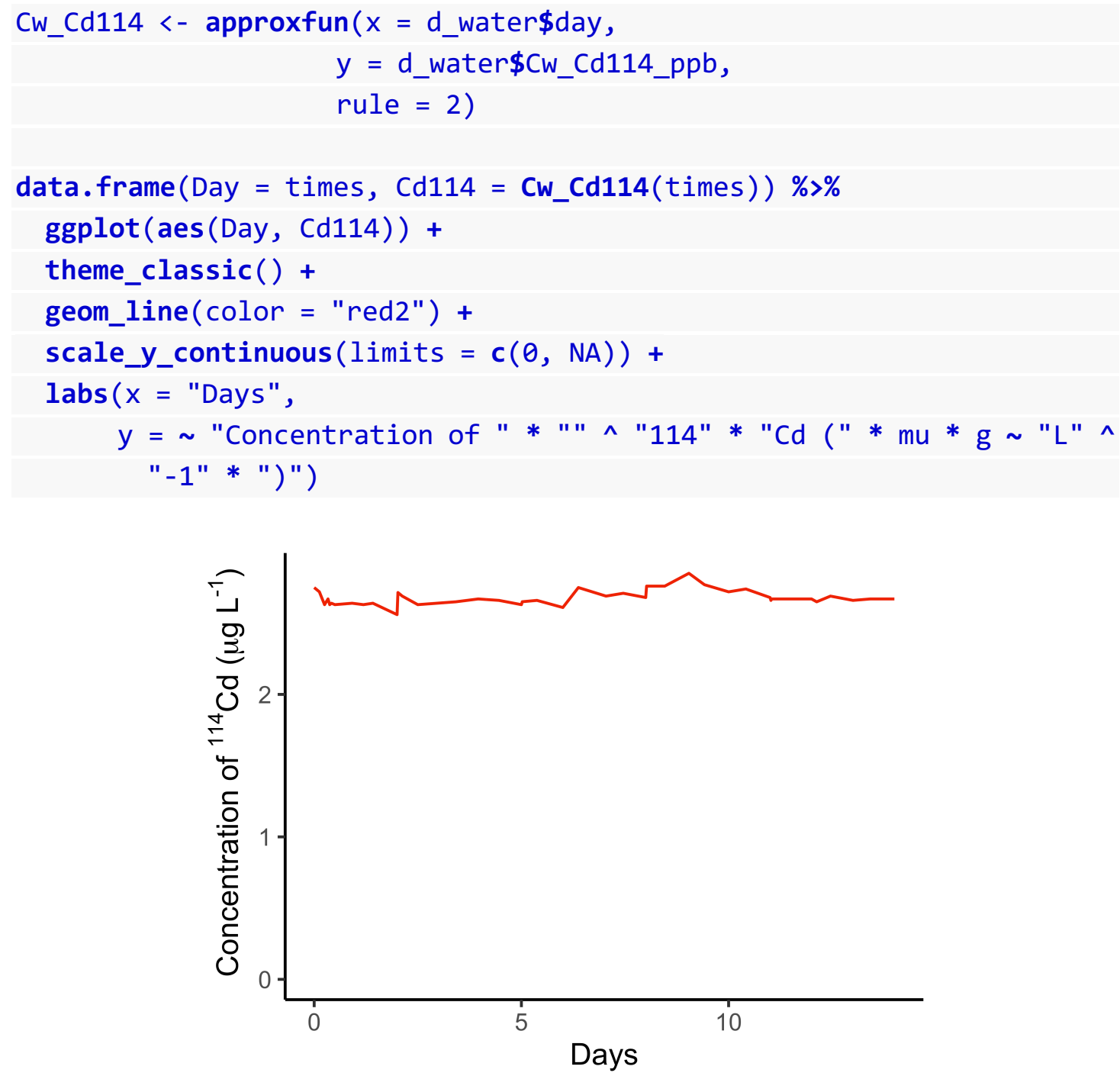


\section{Toxicokinetic (TK) model}

The toxicokinetic model is defined below. The variation of $\mathrm{Cd}$ isotope content in a mussel is the balance between uptake $\left(k_{\text {in }} \cdot C_{\mathrm{w}}\right)$ and elimination $\left(k_{\mathrm{e}} \cdot M_{\text {int }}\right)$ of the isotope. See eqs. 3-5 in the main text for detailed descriptions of the model.

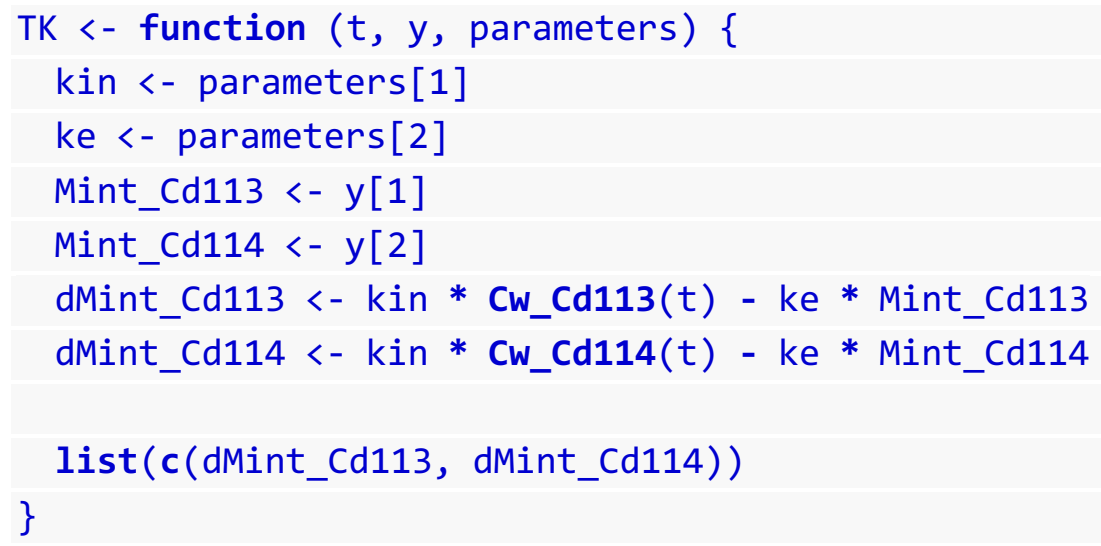

\section{Estimation of the initial parameter values}

An overall rate of $\mathrm{Cd}$ elimination from the mussels can be estimated based on the decrease of ${ }^{113} \mathrm{Cd}$ in mussels during the period between $\mathrm{T} 1$ and $\mathrm{T} 2$ (see the figure below):

$$
k_{\mathrm{e}}=-\frac{\Delta \ln \left(\text { median of new }{ }^{113} \mathrm{Cd}\right)}{\Delta t}
$$

where the numerator is the decrease of the median natural-log transformed ${ }^{113} \mathrm{Cd}$ content, and the denominator $\Delta t$ is the duration of the second stage (i.e., T2 - T1).

The overall $k_{\mathrm{e}}$ was used as the initial value to estimate $k_{\mathrm{e}}$ of individual mussels.

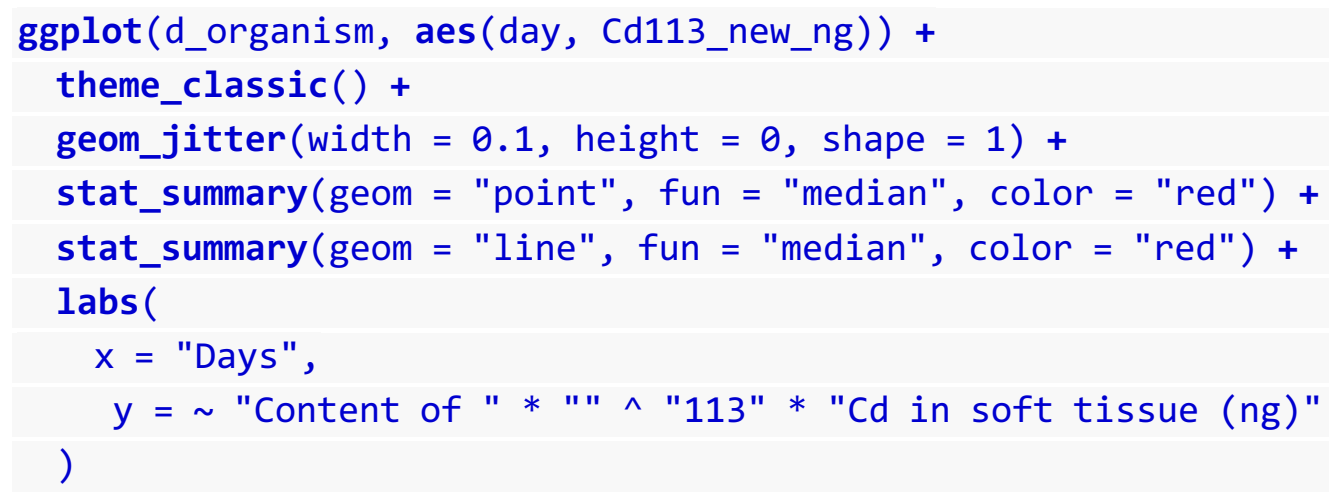



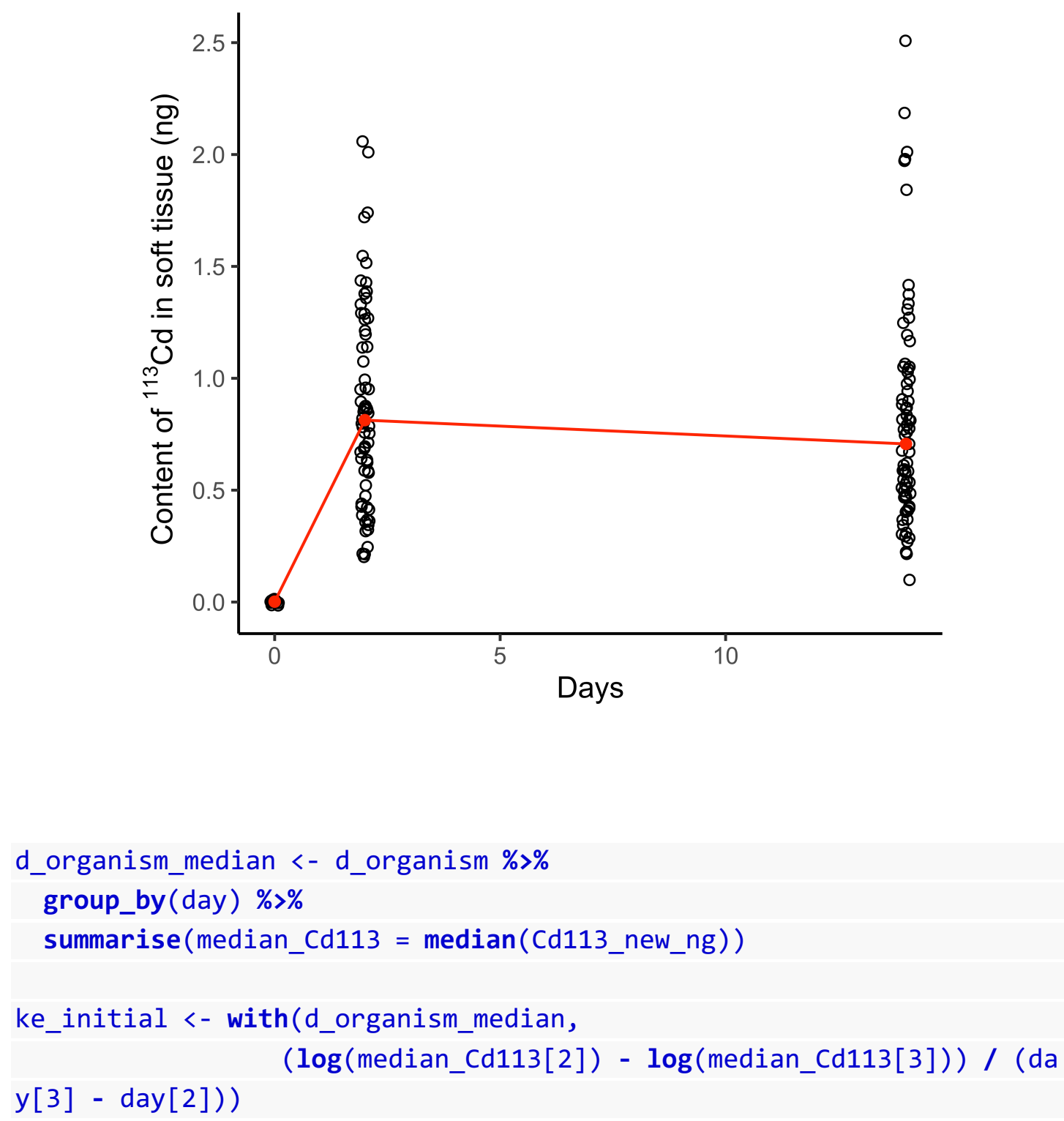


\section{Objective function for fitting the toxicokinetic parameters}

The objective function returns a vector of the weighted residuals, which are the difference between the modeled content of $\mathrm{Cd}$ isotopes and the measured content of $\mathrm{Cd}$ isotopes. The reciprocal of the measured content of $\mathrm{Cd}$ isotopes are used as the weight.

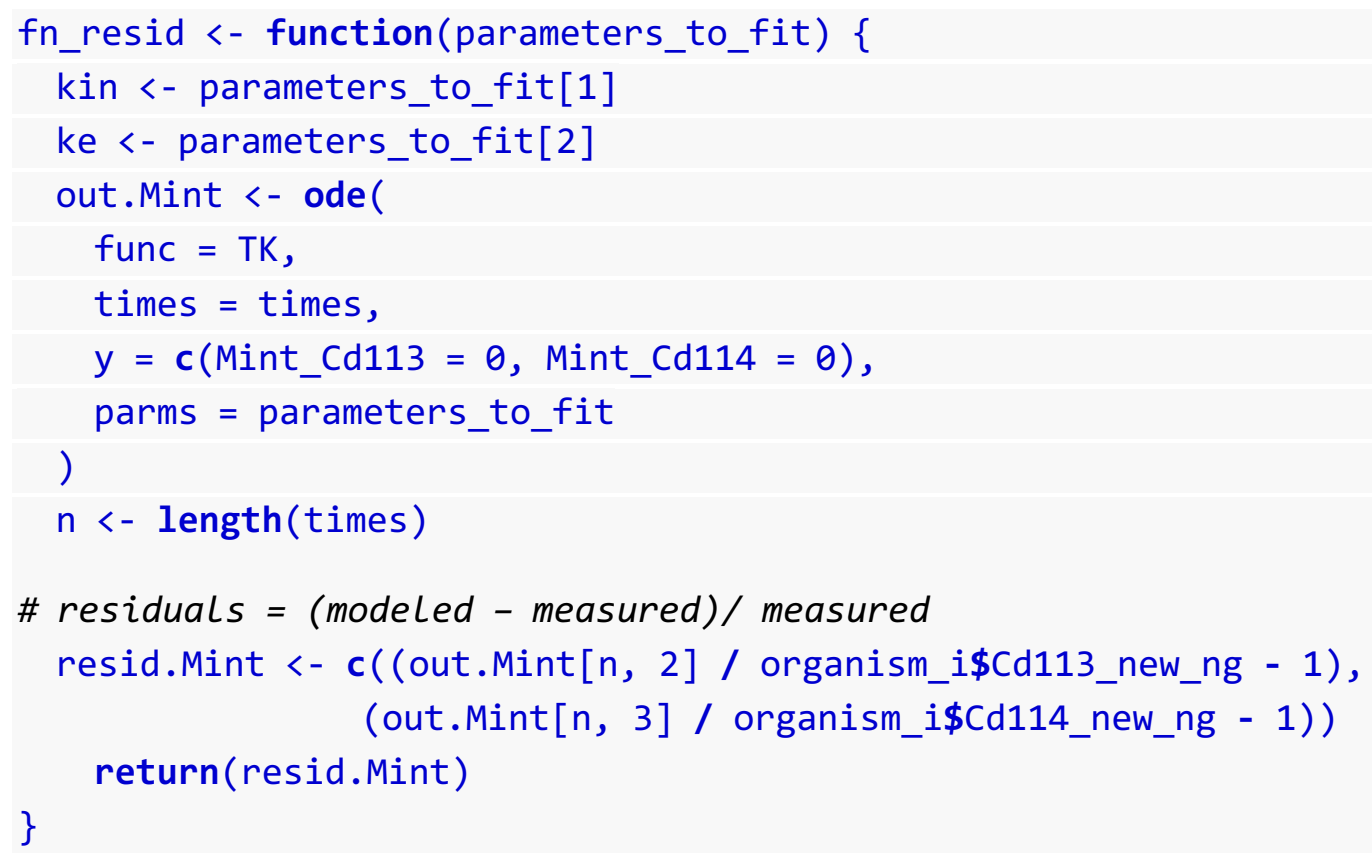

\section{Estimating $k_{\text {in }}$ and $k_{e}$}

Cd isotope contents measured at time T2 are used for estimating $k_{\text {in }}$ and $k_{\mathrm{e}}$.

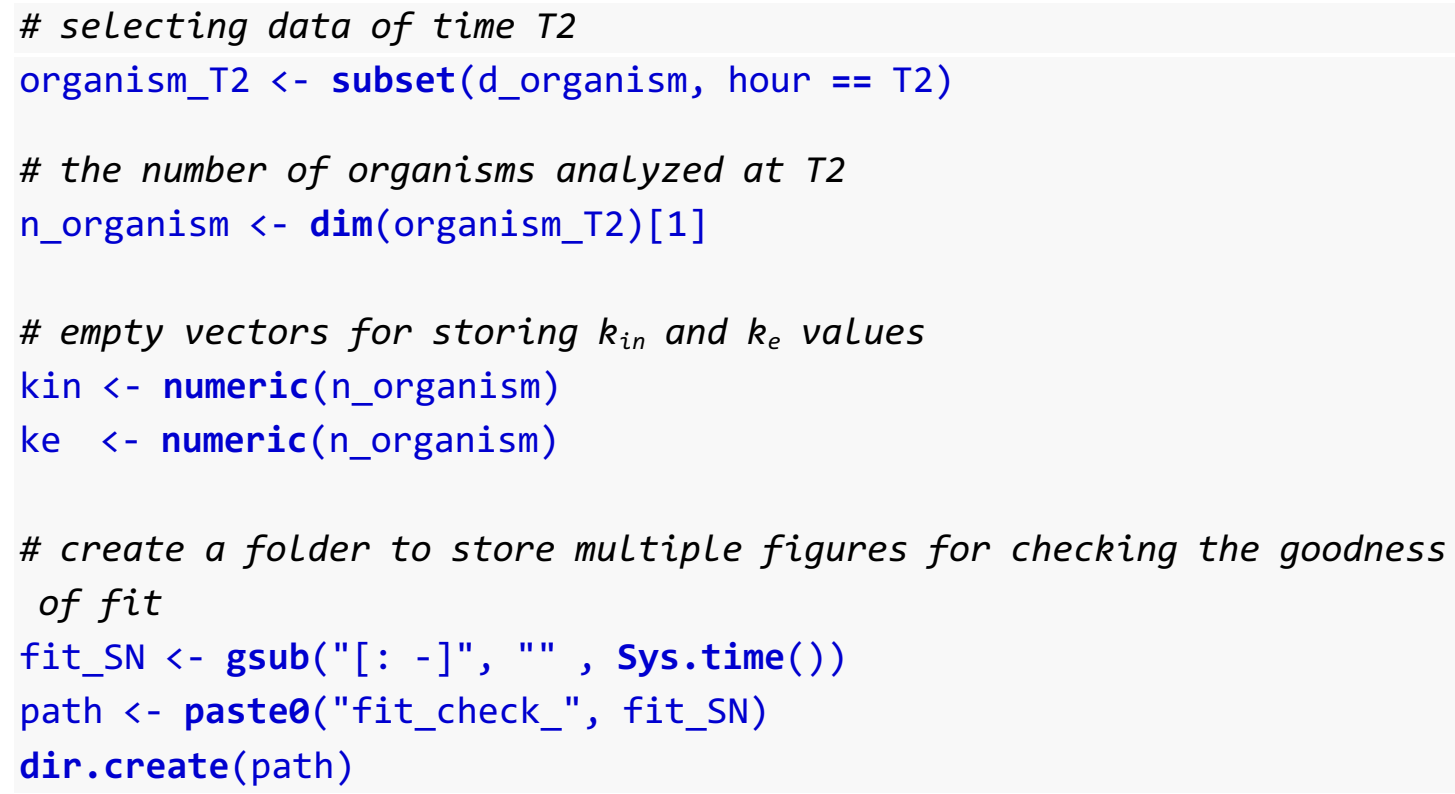


Fitting the values of $k_{\text {in }}$ and $k_{\mathrm{e}}$ for each individual. This step may take $\underline{\mathbf{5} \text { to } 10}$

$\underline{\min }$ to finish.

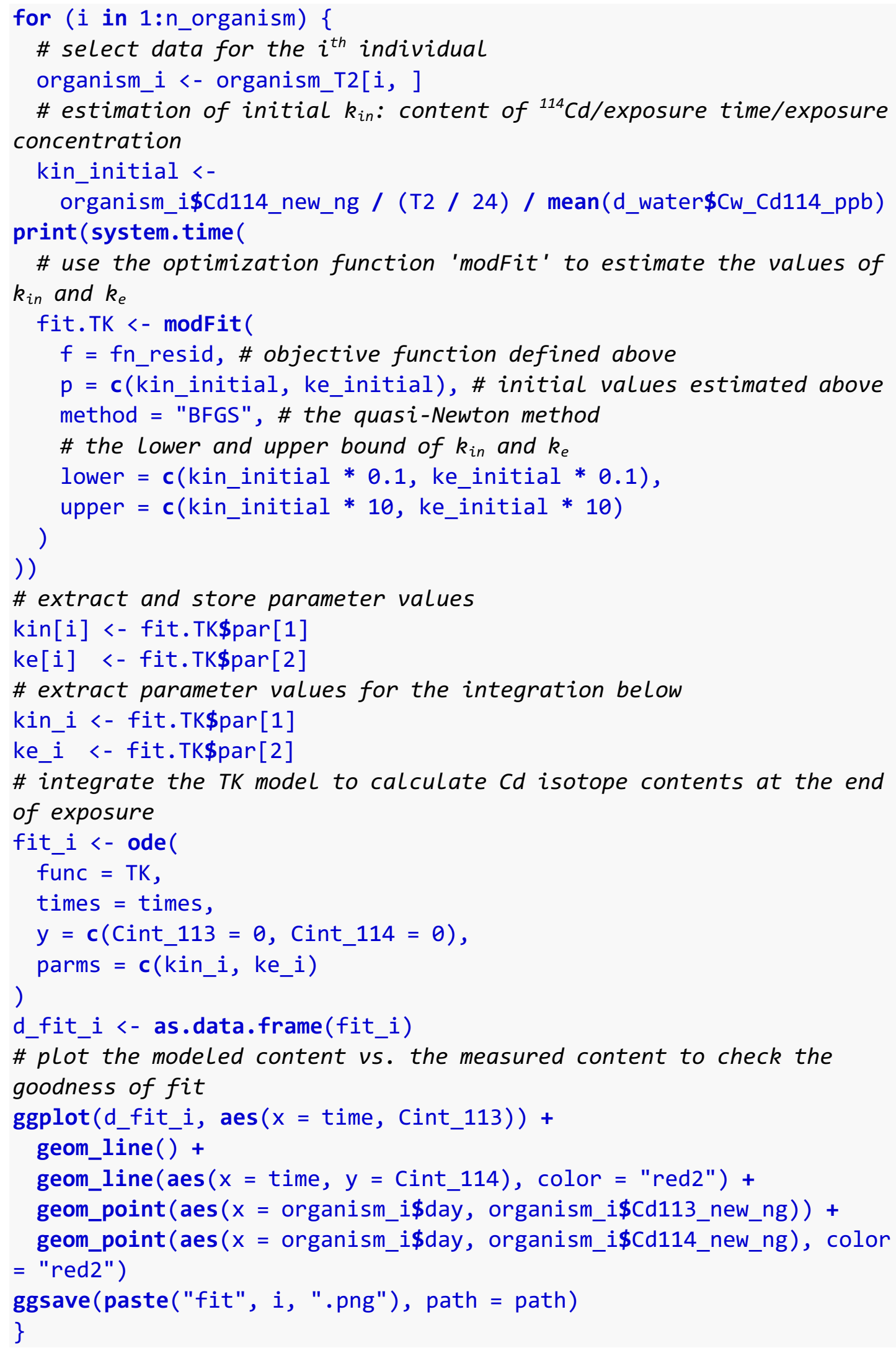




\section{Saving $k_{\mathrm{u}}$ and $k_{\mathrm{e}}$}

The values of $k_{\mathrm{u}}$ and $k_{\mathrm{e}}$ are saved as a *.csv file.

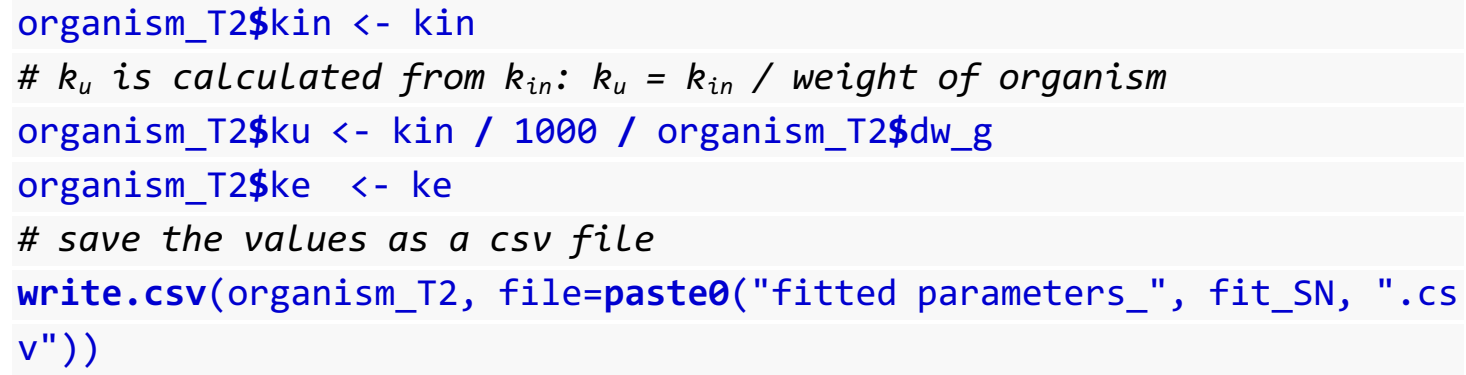

\section{Goodness of fit}

The goodness of fit is visualized by plotting the modeled content vs. the measured content of ${ }^{113} \mathrm{Cd}$ and ${ }^{114} \mathrm{Cd}$ in each organism.

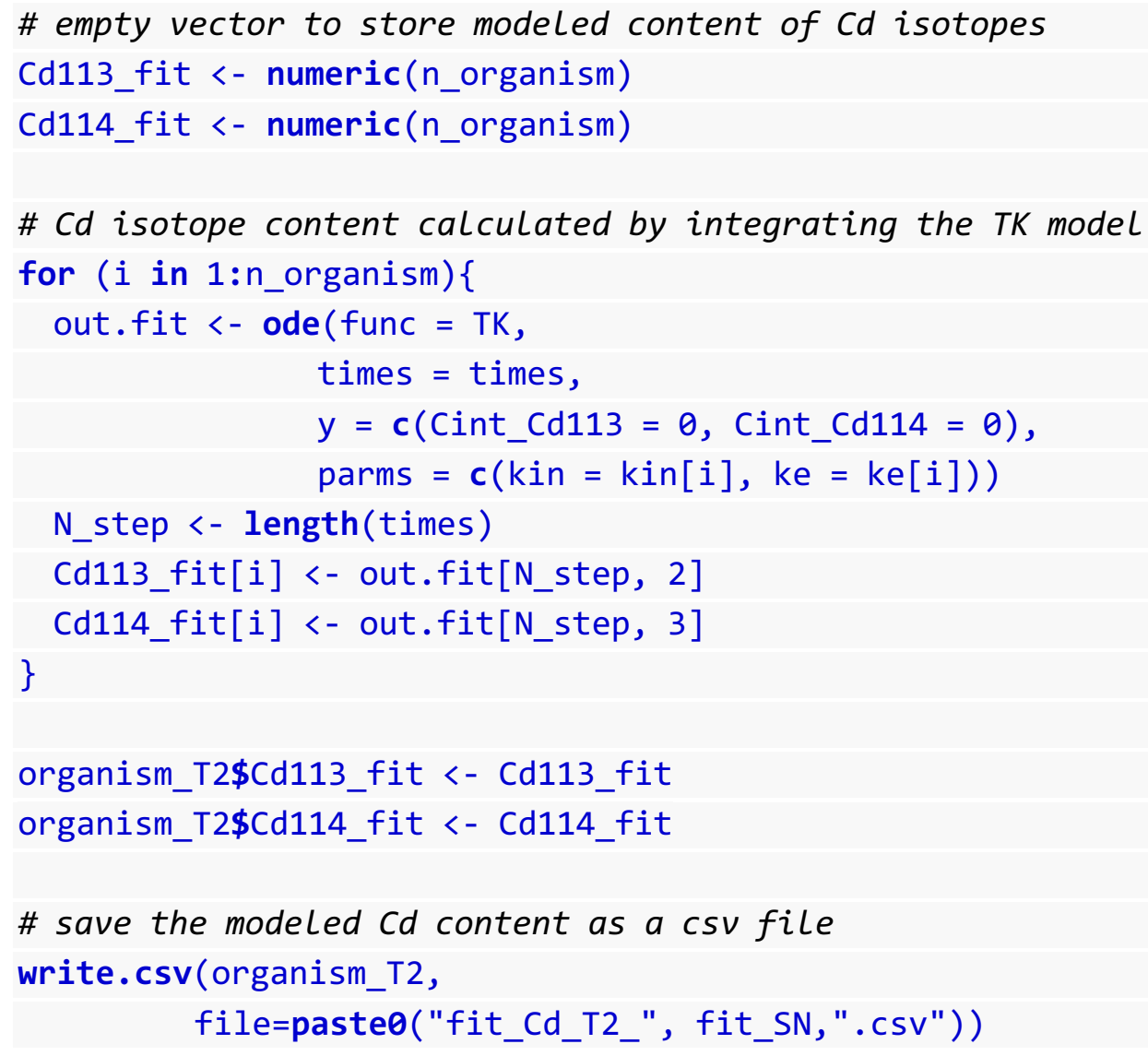




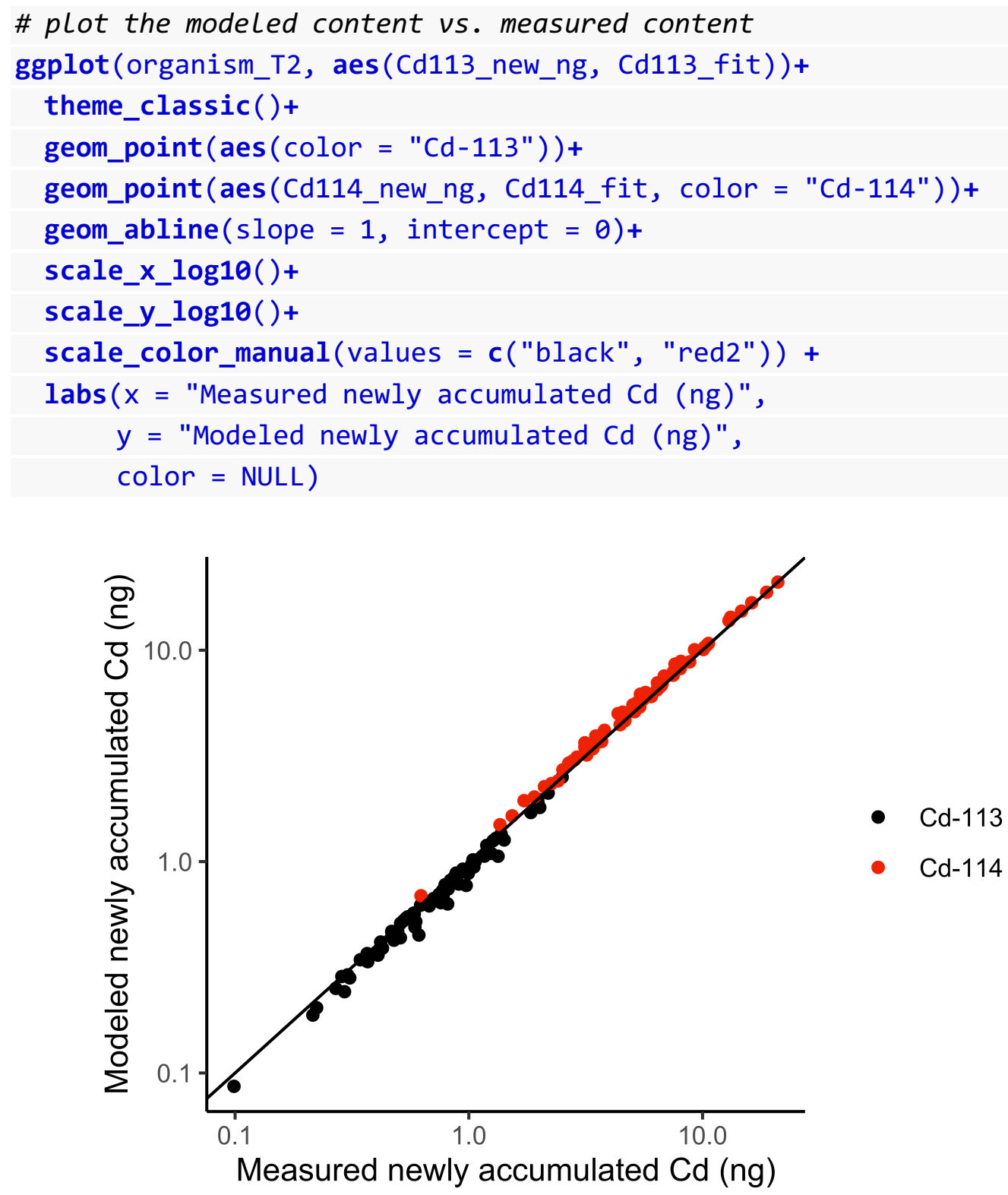




\section{References:}

1. Ju, Y.R., Chen, C.F., Chuang, X.Y., Lim, Y.C., Chen, C.W. and Dong, C.D. 2020. Biometry-dependent metal bioaccumulation in aquaculture shellfishes in southwest Taiwan and consumption risk. Chemosphere 253.

2. Tarique, Q., Burger, J. and Reinfelder, J.R. 2019. Size scaling of contaminant trace metal accumulation in the infaunal marine clam Amiantis umbonella. Archives of Environmental Contamination and Toxicology 77(3), 368-376.

3. Tang, W.L., Evans, D., Kraemer, L. and Zhong, H. 2017. Body size-dependent Cd accumulation in the zebra mussel Dreissena polymorpha from different routes. Chemosphere 168, 825-831.

4. Yap, C., Ismail, A. and Tan, S. 2009. Effect of body size on heavy metal contents and concentrations in green-lipped mussel Perna viridis (Linnaeus) from Malaysian coastal waters. Pertanika Journal of Science and Technology 17(1), 61-68.

5. Pan, K. and Wang, W.-X. 2008. Allometry of cadmium and zinc concentrations and bioaccumulation in the scallop Chlamys nobilis. Marine Ecology Progress Series $365,115-126$.

6. Conti, M.E., Iacobucci, M., Cecchetti, G. and Alimonti, A. 2008. Influence of weight on the content of trace metals in tissues of Mytilus galloprovincialis (Lamarck, 1819): a forecast model. Environmental Monitoring and Assessment 141(1), 27-34.

7. Zhang, L. and Wang, W.-X. 2007. Size-dependence of the potential for metal biomagnification in early life stages of marine fish. Environmental Toxicology and Chemistry 26(4), 787-794.

8. Hedouin, L., Metian, M., Teyssie, J.L., Fowler, S.W., Fichez, R. and Warnau, M. 2006. Allometric relationships in the bioconcentration of heavy metals by the edible tropical clam Gafrarium tumidum. Science of the Total Environment 366(1), 154163.

9. Mubiana, V.K., Vercauteren, K. and Blust, R. 2006. The influence of body size, condition index and tidal exposure on the variability in metal bioaccumulation in Mytilus edulis. Environmental Pollution 144(1), 272-279.

10. Cubadda, F., Conti, M.E. and Campanella, L. 2001. Size-dependent concentrations of trace metals in four Mediterranean gastropods. Chemosphere 45(4), 561-569.

11. Leung, K.M.Y., Morgan, I.J., Wu, R.S.S., Lau, T.C., Svavarsson, J. and Furness, R.W. 2001. Growth rate as a factor confounding the use of the dogwhelk Nucella lapillus as biomonitor of heavy metal contamination. Marine Ecology Progress Series 221, 145-159.

12. Lee, B.-G., Wallace, W., G. and Luoma, S., N. 1998. Uptake and loss kinetics of Cd, $\mathrm{Cr}$ and $\mathrm{Zn}$ in the bivalves Potamocorbula amurensis and Macoma balthica: effects 
of size and salinity. Marine Ecology Progress Series 175, 177-189.

13. Riget, F., Johansen, P. and Asmund, G. 1996. Influence of length on element concentrations in blue mussels (Mytilus edulis). Marine Pollution Bulletin 32(10), 745-751.

14. Catsiki, V.A., Bel, F. and Nicolaidou, A. 1994. Size dependent metal concentrations in two marine gastropod species. Netherland Journal of Aquatic Ecology 28(2), 157-165.

15. Swaileh, K.M. and Adelung, D. 1994. Levels of trace metals and effect of body size on metal content and concentration in Arctica islandica L. (Mollusca: Bivalvia) from Kiel Bay, Western Baltic. Marine Pollution Bulletin 28(8), 500-505.

16. Fischer, H. 1983. Shell weight as an independent variable in relation to cadmium content of mollusks. Marine Ecology Progress Series 12(1), 59-75.

17. Cossa, D., Bourget, E., Pouliot, D., Piuze, J. and Chanut, J.P. 1980. Geographical and seasonal variations in the relationship between trace metal content and body weight in Mytilus edulis. Marine Biology 58(1), 7-14.

18. Cossa, D., Bourget, E. and Piuze, J. 1979. Sexual maturation as a source of variation in the relationship between cadmium concentration and body weight of Mytilus edulis L. Marine Pollution Bulletin 10(6), 174-176.

19. Boyden, C.R. 1977. Effect of size upon metal content of shellfish. Journal of the Marine Biological Association of the United Kingdom 57(3), 675-714.

20. Watling, H.R. and Watling, R.J. 1976. Trace metals in oysters from Knysna estuary. Marine Pollution Bulletin 7(3), 45-48.

21. Boyden, C.R. 1974. Trace element content and body size in molluscs. Nature 251(5473), 311-314.

22. Dang, F. and Wang, W.X. 2012. Why mercury concentration increases with fish size? Biokinetic explanation. Environmental Pollution 163, 192-198.

23. Chong, K. and Wang, W.X. 2001. Comparative studies on the biokinetics of Cd, Cr, and $\mathrm{Zn}$ in the green mussel Perna viridis and the Manila clam Ruditapes philippinarum. Environmental Pollution 115(1), 107-121.

24. Ke, C., Yu, K.N., Lam, P.K.S. and Wang, W.-X. 2000. Uptake and depuration of cesium in the green mussel Perna viridis. Marine Biology 137(4), 567-575.

25. Wang, W.-X. and Dei, R.C.H. 1999. Factors affecting trace element uptake in the black mussel Septifer virgatus. Marine Ecology Progress Series 186, 161-172.

26. Newman, M.C. and Mitz, S.V. 1988. Size dependence of zinc elimination and uptake from water by mosquitofish Gambusia affinis (Baird and Girard). Aquatic Toxicology 12(1), 17-32. 